\title{
A model for RPC detectors operating at high rate
}

\author{
G. Carboni $^{\text {a,* }}{ }^{\text {, G. Collazuol }}{ }^{\text {b }}$, S. De Capua ${ }^{\text {a }}$, D. Domenici ${ }^{\text {a }}$, \\ G. Ganis ${ }^{\text {a }}$, R. Messi ${ }^{\text {a }}$, G. Passaleva ${ }^{\text {b }}$, E. Santovetti ${ }^{\text {a }}$, M. Veltri ${ }^{\text {c }, 1}$ \\ a Università degli Studi di Roma "Tor Vergata" and Sezione INFN Roma 2, Roma, Italy \\ ${ }^{\mathrm{b}}$ Università degli Studi di Firenze and Sezione INFN, Firenze, Italy \\ ${ }^{\mathrm{c}}$ Università degli Studi di Urbino, Urbino, Italy
}

\begin{abstract}
We present a simple model to describe the behavior of Resistive Plate Chambers (RPC) exposed to a high particle flux. We show that the RPC current, $I$, saturates at large flux values and we explain why the dependence of $I$ on the applied voltage $V$ is essentially linear. We show that in the saturated regime the current is controlled by the value of the bulk electrode resitivity, $\rho$, which is directly related to the performance of the detector at high particle rates. Measuring the $I-V_{0}$ curve under these conditions offers a simple and direct method to obtain $\rho$ and to monitor its possible variations.
\end{abstract}

Key words: RPC, detector, current, resistivity, bakelite, high rate PACS: 29.40.Cs

\section{Introduction}

Introduced around 1980, Resistive Plate Chambers (RPC) [1] have recently gained widespread acceptance in High Energy Physics experiments. They have excellent timing performances, and their relatively simple and cheap construction plus the possibility of being tailored to any shape, makes them attractive in particular for large area detectors. Several forthcoming cosmic ray, neutrino and LHC experiments have incorporated these detectors into their designs.

A major drawback of the RPC has been in the past the limited capability to withstand rates larger than a few $\mathrm{Hz} / \mathrm{cm}^{2}$. This limitation was partially overcome by operating them in avalanche mode [2-4], allowing to increase the rate capability up

\footnotetext{
* Corresponding author.

Email address: Giovanni. Carboni@cern.ch (G. Carboni).

1 Also Sezione INFN, Firenze, Italy
} 
to some $\mathrm{kHz} / \mathrm{cm}^{2}$ [5-8]. Once the operating mode is chosen, the most important parameter affecting the rate capability is the volume resistivity of the RPC electrodes. Materials like glasses, with typical resistivities around $10^{12} \Omega \mathrm{cm}$ or more, result in small rate capabilities. Bakelite electrodes, which can be produced with resistivities as low as $10^{9} \Omega \mathrm{cm}$, are used in detectors working at high rate.

Since the rate capability is directly affected by any modification of the RPC resistivity, it is very important to be able to measure this parameter in order to monitor any possible change. In this paper we present a model to describe the behaviour of RPC detectors under high irradiation flux. This model allows the measurement of the resistivity of RPC electrodes in a simple and non destructive way; we discuss here the results obtained in a series of tests performed at the Gamma Irradiation Facilty at CERN, in the framework of the aging studies for the LHCb muon chambers [9].

\section{The model}

\subsection{Operating principle of the RPC}

When a charged particle ionizes the gas of the RPC, an avalanche is formed by multiplication in the gas if the electric field is sufficiently high. The avalanche charge $q$, is related to the ionization charge $q_{i}$, via the gain: $q=G q_{i}$. $G$ depends strongly on the electric field, showing a threshold behavior. When the potential $V_{\text {gap }}$ across the electrodes defining the gas volume (the gas gap) is below some voltage $V_{T}, G$ tends rapidly to unity; when $V_{\text {gap }}>V_{T}$ it increases rapidly with voltage. The value of $V_{T}$ depends on the gap width, the gas composition, and the gas temperature and pressure. In the avalanche mode of operation $G \approx 10^{6}-10^{7}$. Space-charge effects can limit the growth of the avalance charge to a saturation value increasing linearly with $V_{\text {gap }}: q \propto V_{\text {gap }}-V_{T}$. This effect is more evident in gas mixtures containing $\mathrm{SF}_{6}$, where streamer formation is suppressed [10], as in our case.

The current drawn by a detector exposed to a particle flux $\Phi$ (particles/s) is:

$$
I=\Phi q=\Phi G q_{i}
$$

For neutral particles one must take into account their detection efficiency, basically the probability of releasing one or more electrons in the gas. In the case of photons emitted from radioactive sources this efficiency has values between $10^{-3}$ and $10^{-2}[6]$.

In a given detector the electric field, hence the gain and the current, are uniquely determined by $V_{\text {gap }}$. This quantity is not directly measurable, since the voltage drop inside highly resistive electrodes can be quite important if the current flowing through the RPC is large enough. If the detector is connected to an external voltage $V_{0}$ and 
draws a steady current $I$, being exposed to a uniform flux density of particles, the average gap potential is given by Ohm's law:

$$
V_{\mathrm{gap}}=V_{0}-I R
$$

where $R$ is the total resistance of the electrodes. For a typical RPC the bulk resistivity $\rho$ is the dominant contribution to $R$. In that case, for flat electrodes of thickness $d$ and area $S, R=2 \rho(d / S)$.

Fig. 1 shows the avalanche charge measured in an RPC exposed to a very low flux of particles, as a function of $V_{0}$. In this case the voltage drop is negligible and $V_{\text {gap }} \approx V_{0}$. On the same plot we have superimposed the current, to show the expected proportionality with the charge. It is seen that the charge and the current grow linearly with $V_{\text {gap }}$, an indication of the saturation effect.

The $I-V_{\text {gap }}$ curve can be modeled by a linear function with a sharp threshold $V_{T}$ ( $\theta$ is the unit step function)

$$
I\left(V_{\text {gap }}\right)=a \theta\left(V_{\text {gap }}-V_{T}\right)\left(V_{\text {gap }}-V_{T}\right)
$$

folded with a gaussian distribution of $V_{T}$ :

$$
I\left(V_{\text {gap }}\right)=a\left\{\frac{\sigma_{T}}{\sqrt{2 \pi}} e^{-\frac{\left(V_{\text {gap }}-\bar{V}_{T}\right)^{2}}{2 \sigma_{T}^{2}}}+\frac{1}{2}\left(V_{\text {gap }}-\bar{V}_{T}\right)\left[1+\operatorname{erf}\left(\frac{V_{\text {gap }}-\bar{V}_{T}}{\sqrt{2} \sigma_{T}}\right)\right]\right\}
$$

where $\bar{V}_{T}$ is the average value of $V_{T}$ and $\sigma_{T}$ its rms spread, and $a$ is a normalization factor proportional to the particle flux ${ }^{2}$. For $V_{\text {gap }} \gg \bar{V}_{T}$ this function becomes simply $I=a\left(V_{\text {gap }}-\bar{V}_{T}\right)$. A fit of Eq. (3) to the data is presented in Fig. 2, showing an excellent agreement. The relative spread $\sigma_{T} / \bar{V}_{T}$ is typically $3 \%$ to $4 \%$ in the detectors tested. The fact that it varies, albeit slightly, from detector to detector, suggests that geometric effects (e.g. non-uniform gap width) could also contribute to the spread.

\subsection{High-flux conditions}

We consider now a detector exposed to a large and uniform particle flux. From Fig. 1 a typical value for the avalanche charge could be 15 pC. Assuming $2 \mathrm{~mm}$ thick electrodes with $10^{11} \Omega \mathrm{cm}$ resistivity, we can compute from Eq. (1) that $V_{\text {gap }}$ starts being displaced appreciably from $V_{0}$ (say more than $100 \mathrm{~V}$ ) for particle flux densities of $170 \mathrm{~Hz} / \mathrm{cm}^{2}$.

\footnotetext{
$\overline{2}$ It is worth recalling that a possible dark current contribution to $I$ should be subtracted out when interpreting the data.
} 
When the flux increases $V_{\text {gap }}$ shifts more and more towards $V_{T}$. In the limit $\Phi \rightarrow \infty$ the avalanche charge becomes very small to keep the current finite, i.e. we must have $V_{\text {gap }} \rightarrow V_{T}$ to "turn off" the RPC. In this limit we can derive from Eq. (1) the value of the current:

$$
I=I_{\max }=\frac{V_{0}-V_{T}}{R}
$$

Therefore, under high flux conditions, the current will depend linearly on the applied voltage $V_{0}$, via the electrode resistance $R$. The properties of the gas mixture and the gap width only enter via $V_{T}$ and do not affect the slope of the $I-V_{0}$ curve. From the measurement of the $I-V_{0}$ characteristic curve in high radiation conditions one can extract $R$ and can easily monitor its variations. We stress here that the linearity of Eq. (4) is based only on the general assumption of the presence of a sharp threshold effect in the avalanche evolution. In particular, it has no relation with the linear dependence of the avalanche charge on $V_{\text {gap }}$ (Fig. 1), and also holds in a regime of non saturated avalanche.

Eq. (4) can be generalized for arbitrary flux values: using Eq. (1) and Eq. (2), one has for $V_{0}>V_{T}$ :

$$
I=\left(\frac{a R}{1+a R}\right) \frac{V_{0}-V_{T}}{R}=\left(\frac{X}{1+X}\right) \frac{V_{0}-V_{T}}{R}=\frac{V_{0}-V_{T}}{R_{\mathrm{eff}}}
$$

It will be noticed that the parameter $X=a R$ controls the deviations from the simple proportionality between the current and the flux. Since $a \propto \Phi$, this causes the current to saturate at $I_{\max }$ for large values of the flux. The saturation is most readily achieved for detectors of large resistance, where the internal voltage drop IR may attain values of hundreds of volts at relatively small flux.

By including in Eq. (5) the spread in $V_{T}$ we obtain the equivalent of Eq. (3) with the substitutions $V_{\text {gap }} \rightarrow V_{0}$ and $a \rightarrow R^{-1} X /(1+X)$. This formula will be used in the rest of the paper to describe the $I-V_{0}$ curve, and in particular to extract $R_{\text {eff }}$ from its slope.

To compute the true resistance, $R=R_{\mathrm{eff}} X /(1+X)$, the value of $X$ is also necessary. If $X$ is known a priori to be large, the correction can be neglected. Otherwise, $X$ must be obtained by fitting the current (or $R_{\text {eff }}$ ) at different values of the flux. It is important to underline that, if $X$ is large, a simple estimate can be sufficient: the correction introduces in fact only a very small uncertainty on $R$. So the method works better under high-flux conditions and for large resistance values, since in this case $X \gg 1$.

This model also makes a definite prediction about the dependence of the current on the temperature via Eq. (5), giving a simple way to correct experimental data for temperature effects. In the case of RPCs with bakelite electrodes the dominant effect on $I$ comes from the exponential dependence of the resistivity on the 
temperature. The corrections due to $V_{T}$ variations are expected to be negligible in comparison.

\section{Comparison with data}

\subsection{Experimental setup}

The tests described in the following have been performed at the Gamma Irradiation Facility (GIF) at CERN. The GIF is a test area where particle detectors are exposed to an adjustable photon flux from an intense radioactive source. The active element is ${ }^{137} \mathrm{Cs}$ ( $30.2 \mathrm{y}$ half-life) which emits $662 \mathrm{keV}$ photons with a $85 \%$ branching ratio. The activity was measured to be $740 \mathrm{GBq}$ on 5 March 1997. A high-energy particle beam from the SPS accelerator traverses the test area, so that various measurements can be performed on detectors in presence of the high background flux of photons from the source. In our case the source was also used to perform an accelerated aging test of the detectors. Beyond a precisely shaped collimator, the source incorporates a thin lens-shaped lead filter that renders the outcoming flux uniform on a vertical plane. A system of remotely controlled filters allow to vary the photon rate by four orders of magnitude. A complete description of the GIF is given in [11].

In our tests two equal RPC detectors were operated in the GIF area. The detectors $\left(50 \times 50 \mathrm{~cm}^{2}\right.$ with a $2 \mathrm{~mm}$ gas gap) were built using bakelite plates ( $2 \mathrm{~mm}$ thick) of nominal resistivity $\approx 10^{10} \Omega \mathrm{cm}$, and treated internally with linseed oil. The plates were supported by a peripheral polycarbonate frame, and by round spacers arranged in a square grid of $10 \mathrm{~cm}$ side. Externally the plates were painted with graphite in order to provide the conductive surfaces where the high voltage was applied.

Both detectors were operated with the same gas mixture, normally $95 \% \mathrm{C}_{2} \mathrm{H}_{2} \mathrm{~F}_{4}$, $4 \% \mathrm{i}-\mathrm{C}_{4} \mathrm{H}_{10}$ and $1 \% \mathrm{SF}_{6}$. The gas mixture was adjusted by mass flowmeters, controlled by a PC. High-voltage $V_{0}$, current and temperature were continuously monitored and recorded during all the test period (more than one year).

The test setup is schematically shown in Fig. 3. Normally during the aging test one detector (A) was placed in position 1, very close to the source and almost continuously exposed to radiation, whereas the second (B) was placed far from the source (position 2), to serve as a reference.

Position 3 was used to perform efficiency measurements with the particle beam. In this case the signals from the RPCs were read out on $3 \mathrm{~cm}$ wide strips using fast electronics. A telescope of scintillator counters, also shown in Fig. 3, provided the trigger and a hodoscope measured the particle position $(x-y)$ with an accuracy better than $1 \mathrm{~cm}$. At the minimum source attenuation the measured flux density in 
Position 3 was about $1 \mathrm{kHz} / \mathrm{cm}^{2}[12]$.

\subsection{Current versus flux}

In comparing the model with the data it is very important to know accurately the relative absorption factors (Abs) of the various GIF filters. The nominal values are correct only for the direct $(662 \mathrm{keV})$ radiation. The total intensity (direct plus diffused radiation) is not linear in 1/Abs but rather follows a power law $\left(\Phi \propto \mathrm{Abs}^{-\beta}\right)$. From the ionization chamber data [11] one obtains $\beta=0.8$. Since the exponent could depend on the effective energy spectrum of the photons for the detector under study, we calibrated the filters directly measuring the current of an RPC of low resistivity $\left(\rho \approx 10^{10} \Omega \mathrm{cm}\right.$ ), placed at about $1.4 \mathrm{~m}$ from the source. In that case the current was simply proportional to the flux, since $X \ll 1$. Our measurement gave $\beta=0.70 \pm 0.01$ (see Fig. 4).

The study of the current dependence upon flux has been performed by placing the detectors close to the source, at about $55 \mathrm{~cm}$ distance (position 1, Fig. 3). Fig. 5 shows the current of RPC A, measured at several filter absorptions, for two values of the applied voltage $V_{0}(10000$ and $10800 \mathrm{~V})$ well above the threshold voltage $V_{T}$. The nominal filter absorption of the source was varied between 1 and $10^{4}$. Saturation is evident already at low flux values $\left(\mathrm{Abs}^{-1} \approx 10^{-3}\right)$. Fitting Eq. (5) to the data was done using the relationship $X(\mathrm{Abs})=X(\mathrm{Abs}=1) \mathrm{Abs}^{-0.7}$, with $X(\mathrm{Abs}=1)$ as a free parameter. We obtained $X(\mathrm{Abs}=1)=49.8 \pm 8.9$ with very good agreement over almost three orders of magnitude on the flux. The corresponding $98 \%$ saturation implies only a minor correction to the resistance value.

As expected, $X$ was smaller at larger distances from the source, since it decreases proportionally to the flux. Going from $\approx 55 \mathrm{~cm}$ to $\approx 140 \mathrm{~cm}$ the saturation corrections became as low as $75 \%$. For that reason, the resistivity measurements were normally taken at the minimum possible distance, about $55 \mathrm{~cm}$.

\subsection{Resistance measurements}

Typical $I-V_{0}$ curves taken for two different GIF absorption factors (Abs=1 and Abs=10) are shown in Fig. 6 for the two detectors under test ${ }^{3}$. A perfect linearity is observed. From the slopes it appears that the two detectors have slightly different resistivities, both around $6 \cdot 10^{11} \Omega \mathrm{cm}$. The modest increase in the current passing from $A b s=10$ to $A b s=1$ (corresponding to a factor 5 increase of the flux) is an effect of the large saturation, about $98 \%$ at $A b s=1$.

$\overline{3}$ During this measurement, also detector B was placed close to the source, position 1 in Fig. 3. 
The values of $X$ were obtained, as said previously, by measuring the current at a fixed $V_{0}$ value for several different photon intensities. Since $X$ is proportional to the resistance $R$, its value could change considerably, in particular according to the temperature variations. It was however not necessary to repeat frequently the measurements of $X$, since we could simply scale its value proportionally to $R$ (or to $R_{\text {eff }}$ to a first approximation). During all the period of the test, $X$ (measured at the smallest absorption factor Abs=1) ranged from about 7 to more than 50 .

It is interesting to use the values of $R$ obtained by the present method to interpret the efficiency curves of RPCs for minimum ionizing particles (MIPs). The assumption that also the detection efficiency of the detector depends solely on $V_{\text {gap }}$ has already been used to extract $R$ from efficiency curves [12-14]. Measuring the MIP efficiency vs. $V_{0}$ in presence of a large photon background rate from the GIF, we obtained several curves (Fig. 7, left) for different absorption factors. When the efficiency is plotted vs. $V_{\text {gap }}=V_{0}-I R$, using the value of $R=31.6 \mathrm{M} \Omega$ determined by our model, the curves coalesce as expected (Fig. 7, right).

\subsection{Temperature effects}

As discussed in Sect. 2.2 the temperature dependence of the RPC resistance should be the same as for bakelite bulk resistivity. i.e. it should have the form:

$$
R \approx R_{20} \exp \left[\alpha\left(\theta_{20}-\theta\right)\right]
$$

where $\theta$ is the temperature in ${ }^{\circ} \mathrm{C}$ and $\theta_{20}=20{ }^{\circ} \mathrm{C}$. Measuring directly the bulk resistivity on three different bakelite samples we obtained $\alpha=0.12 \pm 0.01$.

In Fig. 8, the measurements of the resistance of RPC A and B are shown as a function of the temperature. An exponential fit of these data gives a temperature coefficient $\langle\alpha\rangle=0.126 \pm 0.008$ in nice agreement with that of the bakelite itself, thus confirming our interpretation of temperature effects.

As observed above, Eq. (5) allows to correct the RPC current measurements for temperature effects, that are introduced through $R$, allowing an unambiguous normalization of the current itself, for example at $20^{\circ} \mathrm{C}$. This is shown in Fig. 9, where the effect of 24-h temperature oscillations is almost completely corrected for.

Finally Fig. 10 presents the corrected current (normalized at $20{ }^{\circ} \mathrm{C}$ ) of RPC A as measured during the 2001 irradiation test. Apart from some jumps, related to occasional changes in the high voltage, changes in the gas composition and to the insertion or removal of other detectors between the GIF and the RPC, a steady exponential decrease of the current is observed. This is attributed to a corresponding increase of the detector resistivity of almost one order of magnitude, which seems to be the main effect of RPC aging. A complete analysis of this effect is in progress. 


\section{Conclusions}

We have developed a model to study the RPC behavior under high irradiation. The model allows to obtain the detector resistance under operating conditions, and to correct unambiguously the current for temperature effects.

Acknowledgments We would like to thank A. Bizzeti and V. Souvorov for valuable help during the preparation and running of these tests.

\section{References}

[1] R. Santonico and R. Cardarelli, Nucl. Instr. and Meth. in Phys. Res. A 187 (1981) 377.

[2] R. Cardarelli, A. Di Ciaccio and R. Santonico, Nucl. Instr. and Meth. in Phys. Res. A 333 (1993) 399.

[3] C. Bacci et al., Nucl. Instr. and Meth. in Phys. Res. A352 (1995) 552.

[4] R. Cardarelli, V. Makeev, R. Santonico, Nucl. Instr. and Meth. in Phys. Res. A 382 (1996) 470.

[5] R. Cardarelli, Scientifica Acta VIII (Univ. Pavia), 3 (1993) 159.

[6] M. Adinolfi et al., Nucl. Instr. and Meth. in Phys. Res. A456 (2000) 95.

[7] R. Arnaldi et al., Nucl. Instr. and Meth. in Phys. Res. A456 (2000) 72.

[8] M. Ćwiok et al., Nucl. Instr. and Meth. in Phys. Res. A456 (2000) 87

[9] LHCb Collaboration, LHCb Muon System Technical Design Report, CERN/LHCC 2001-010, 2001.

[10] P. Camarri et al. Nucl. Instr. and Meth. in Phys. Res. A 414 (1998) 317.

[11] S. Agosteo et al., Nucl. Instr. and Meth. in Phys. Res. A 452 (2000) 94.

[12] G. Ganis et al., Proceedings of the "VI Workshop on RPC and Related Detectors", Coimbra, November 2001; physics/0210045

[13] G. Aielli et al., Nucl. Instr. and Meth. in Phys. Res. A 456 (2000) 82.

[14] G. Passaleva et al., Proceedings of the "International Workshop on Aging Phenomena in Gaseous Detectors”, DESY, October 2001. 


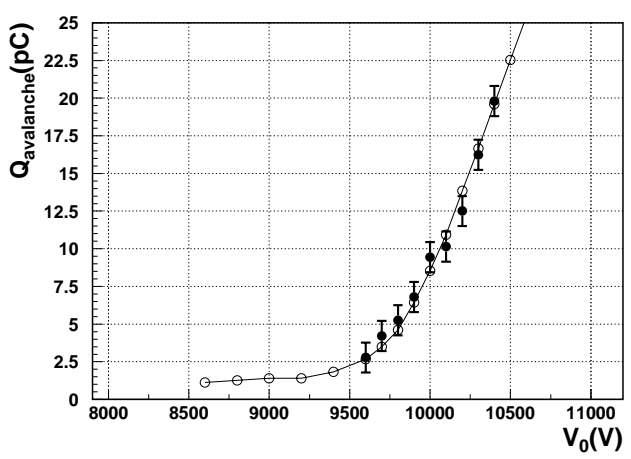

Fig. 1. Solid circles: avalanche charge vs. $V_{0}$, measured with cosmic rays. Open circles: current (arbitrary scale) measured with low particle flux. In both cases $V_{\text {gap }} \approx V_{0}$ (see text). RPC operated with $\mathrm{C}_{2} \mathrm{H}_{2} \mathrm{~F}_{4}, \mathrm{i}-\mathrm{C}_{4} \mathrm{H}_{10}$ and $\mathrm{SF}_{6}$ mixture in 95:4:1 proportion.

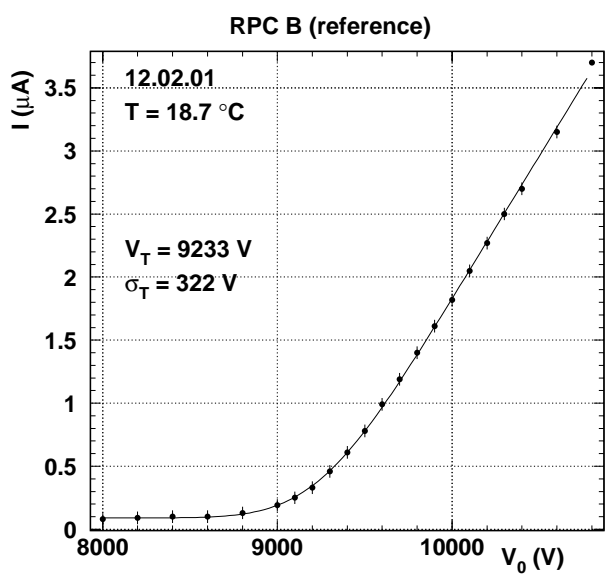

Fig. 2. RPC current vs. $V_{0}$ measured with low particle flux $\left(V_{\text {gap }} \approx V_{0}\right)$ and fitted with the function described in the text. 


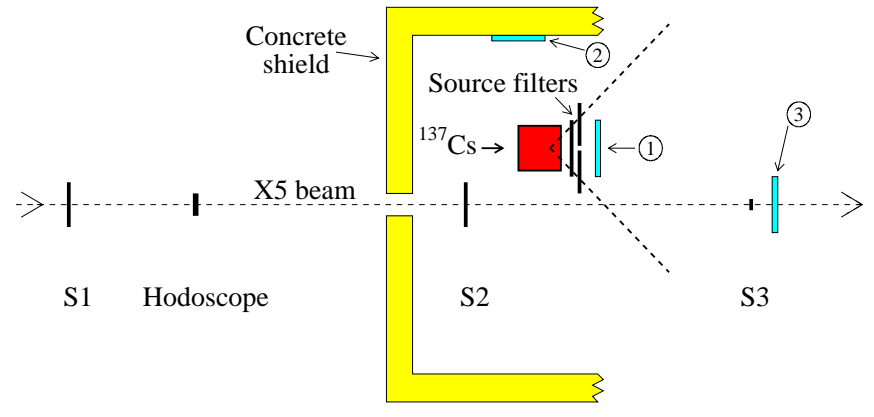

Fig. 3. Schematic view of the test setup (not to scale). The positions of the RPCs corresponding to the various measurements are indicated (1-3). The distance of the RPC from the source in position 1 was about $55 \mathrm{~cm}$, and in position 3 about $140 \mathrm{~cm}$. The scintillator counters (S1-S3 and the Hodoscope) were used for measuring the RPC efficiency with the particle beam.

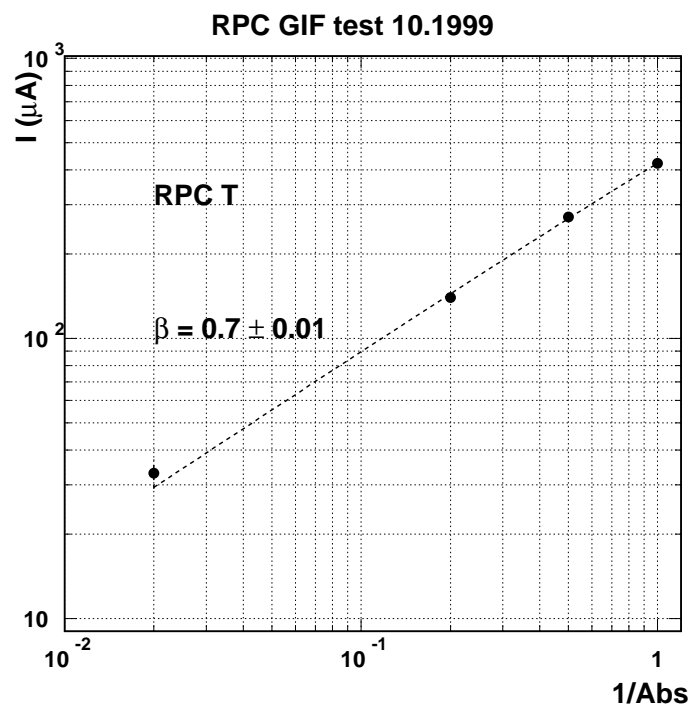

Fig. 4. Current for a detector of low resistivity at fixed $V_{0}(10200 \mathrm{~V})$ plotted against the inverse of the nominal source absorption factor (see text). The behavior shows negligible saturation. The fit gives $\beta=0.70 \pm 0.01$. 


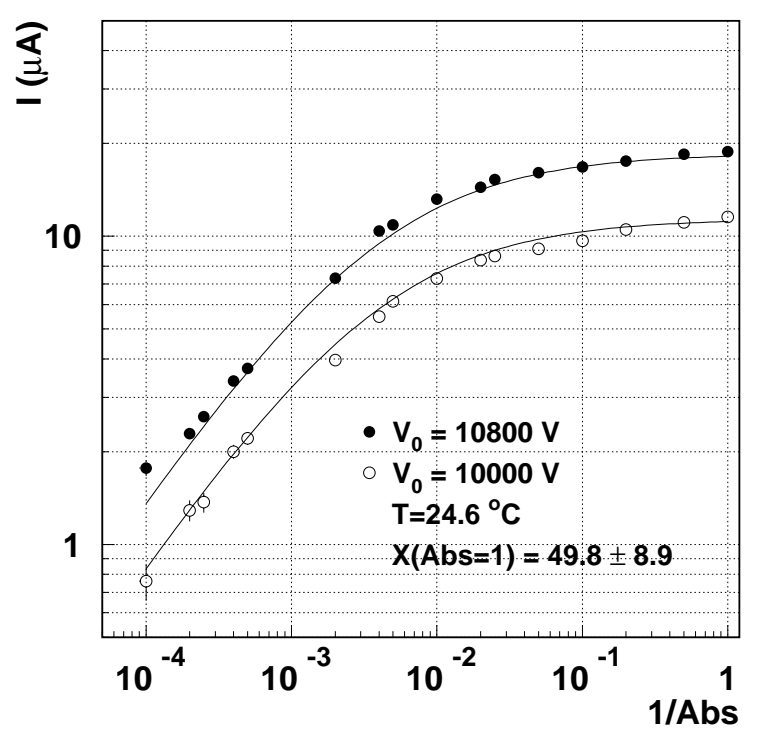

Fig. 5. RPC current measured at fixed $V_{0}(10800 \mathrm{~V}$, solid circles; $10000 \mathrm{~V}$, open circles), plotted against the inverse of the nominal source absorption (see text). The curves are the predictions of the model.
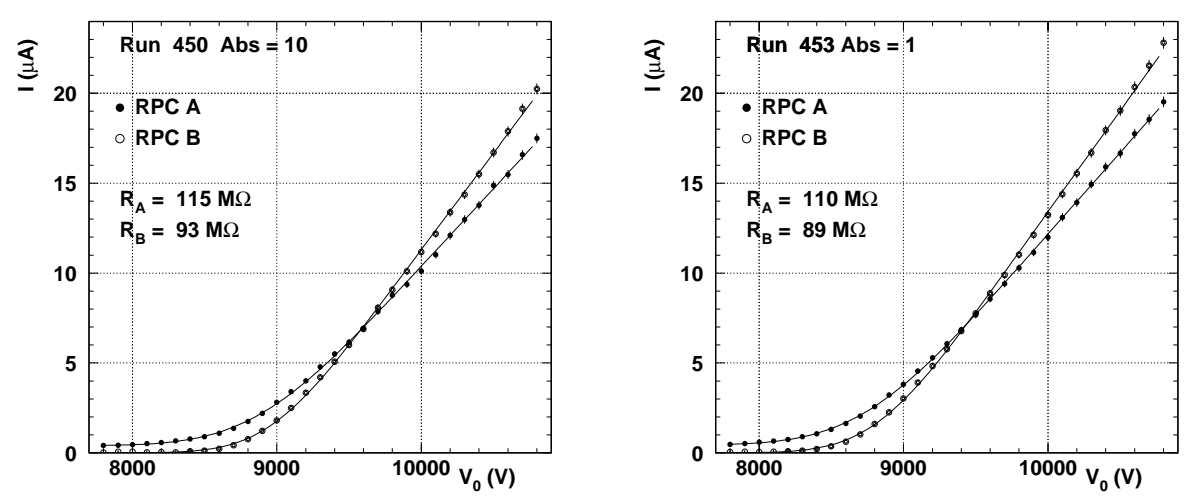

Fig. 6. Current vs. $V_{0}$ for RPC A (solid circles) and B (open circles) for two source nominal absorptions factors (10 left; 1 right). The effective resistances $R_{\mathrm{eff}}$ as obtained from the slopes are also shown. In this case the saturation was $98 \%$. 

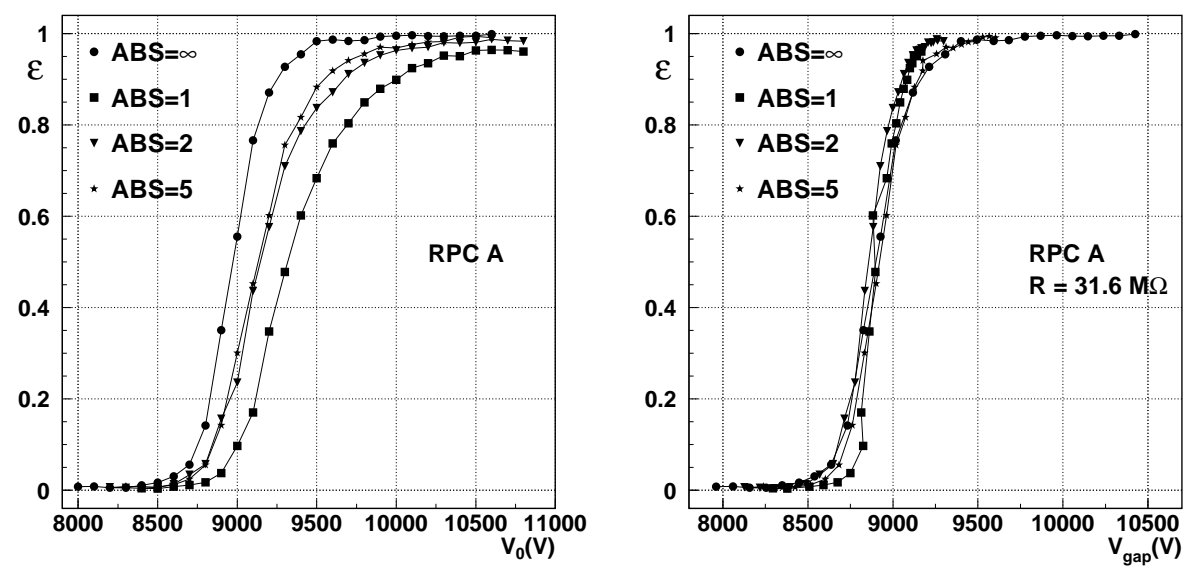

Fig. 7. Efficiency for MIPs vs. $V_{0}$ for RPC A (left), measured in presence of different photon fluxes from the source (the nominal filter absorption factors are indicated). On the right the same data are plotted vs. $V_{\text {gap }}=V_{0}-I R$ using the value of $R$ determined by the model.

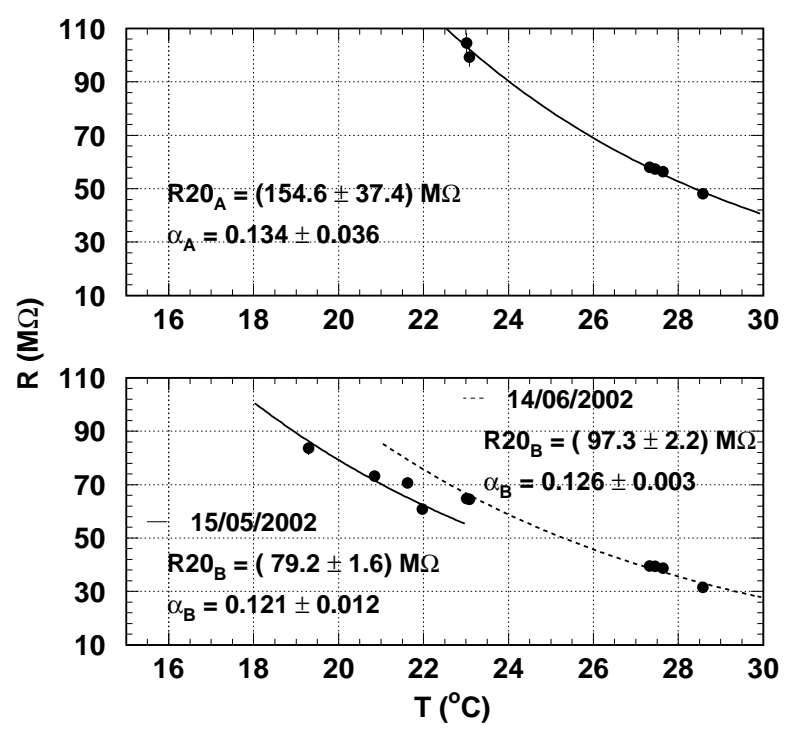

Fig. 8. Resistances of RPC A and B plotted versus temperature. The temperature coefficient $\alpha$ is fitted using the exponential dependence discussed in the text. 

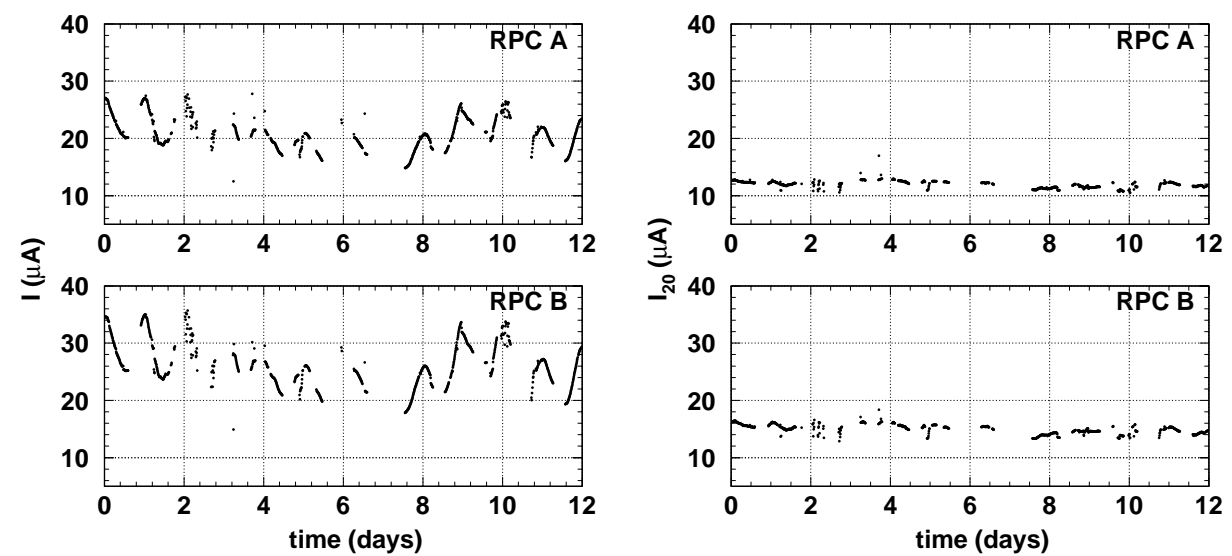

Fig. 9. Time evolution of currents for RPC A and B under GIF irradiation. Raw currents are on the left, showing typical daily oscillations. The corrected currents at $20^{\circ} \mathrm{C}$ are on the right.

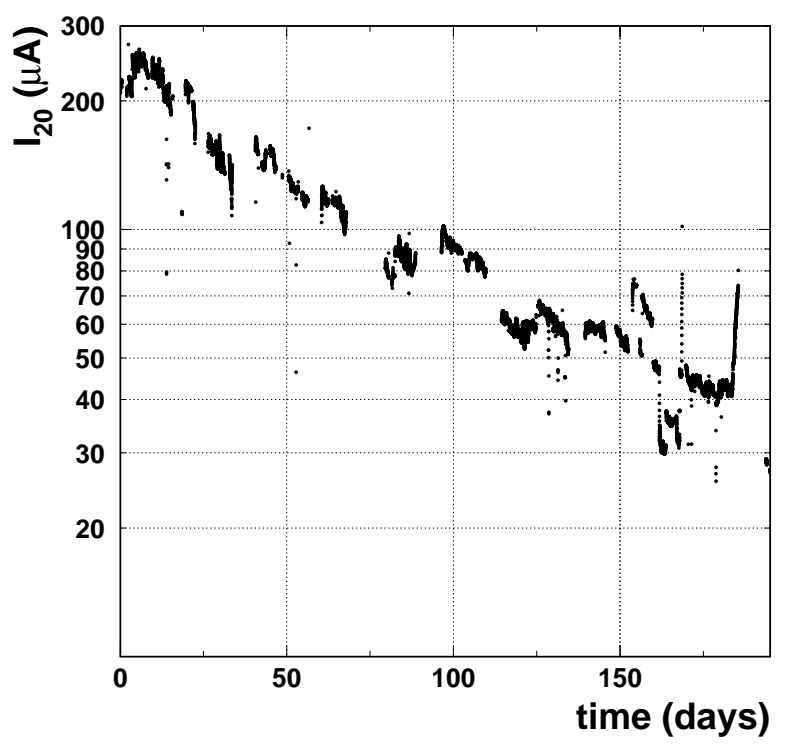

Fig. 10. Current for RPC A corrected for temperature (see text) plotted versus time. The detector was placed at about $55 \mathrm{~cm}$ from the source. Deviations from the exponential decrease are due to changes in the $\mathrm{HV}$, gas mixture, insertion or removal of other detectors in front of the source. 